\title{
On the influence of cyclic variability on surface noise contribution analysis of internal combustion engines
}

\author{
A. Acri ${ }^{\text {a,b,* }}$, E. Nijman ${ }^{\mathrm{a}}$, M. Klanner ${ }^{\mathrm{c}}$, G. Offner ${ }^{\mathrm{d}}$, R. Corradi ${ }^{\mathrm{b}}$

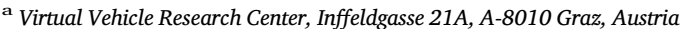 \\ b Dipartimento di Ingegneria Meccanica, Politecnico di Milano, Italy

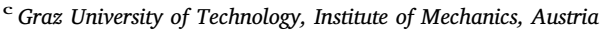 \\ ${ }^{\mathrm{d}}$ AVL List GmbH, Austria
}

\begin{abstract}
Practical mechanical systems often operate with some degree of uncertainty. The uncertainties can result from poorly known or variable parameters, from uncertain inputs or from rapidly changing forcing that can be best described in a stochastic framework. In automotive applications, cylinder pressure variability is one of the uncertain parameters that engineers have to deal with when designing and analyzing internal combustion en-gines. The characterization of acoustic radiation patterns of internal combustion engines is a challenging task required for the purpose of effective noise reduction. In this paper the influence of cylinder pressure cyclic variability on the assessment and ranking of the different radiating engine surfaces is investigated. A surface contribution analysis (SCA) within a wave based method (WBM) framework is adopted for the assessment of noise radiated from different vibrating surfaces of an engine structure. The method adopted consists in the decomposition of the boundary conditions of the WBM model, assuming the linearity of the vibrational problem associated to the generation of the vibrations on the structure surface, for which the superposition principle is valid. In order to investigate the cyclic variability of cylinder pressure, a Monte Carlo approach is adopted. Starting from measured cylinder pressures that exhibits cyclic variability, random Gaussian distribution of the equivalent force applied on the piston is generated. The results obtained from this analysis are used to derive correlations between cyclic variability and statistical distribution of the results. The statistical information derived can be used to advance the knowledge of the WBM and SCA applications when uncertain inputs are considered.
\end{abstract}

\section{Introduction}

Together with the reduction of fuel consumption and exhaust pollution, noise reduction is one of the main topics of interest in internal combustion engine development, because of the more and more strict noise legislation and the increasing customer demands. Engine noise can be separated into structure-borne noise and air borne noise. Structure-borne noise is generated from combustion and mechanical phenomena that occur inside the engine, it is transmitted through the engine structure and it is radiated from the external surface of the engine. Conversely, air borne noise is directly generated into the air in correspondence of intake and exhaust systems. The air borne noise can be significantly reduced adopting air intake and exhaust mufflers, therefore, surface radiated noise becomes the predominant acoustic issue. Covers, such as oil pans, valve covers and front gear covers, are important engine components used to contain liquids (oil and/or water) and, in many applications, also to reduce the noise emission of the internal components. It has been shown that most of the noise is radiated from covers and an optimal cover design can considerably improve the noise emission $[1,2]$. In order to understand where and how to improve the external covers and engine surfaces design, it is neces-sary to analyze and rank the different engine surfaces in terms of emitted noise. Experimentally, several methods can be used to rank the different radiating surfaces: Lead covering (also called shielding tech-nique), surface vibrations technique, acoustic intensity technique and acoustic holography [3-5]. To reduce design and development timing and costs, computer-aided-engineering (CAE) methods are extensively used. In industrial applications the finite elements method (FEM) and the boundary element method (BEM) are the most widely used methods to predict the acoustic field in interior and exterior radiation problems.

* Corresponding author at: Virtual Vehicle Research Center, Inffeldgasse 21A, A-8010 Graz, Austria.

E-mail addresses: Antonio.Acri@v2c2.at, antonio.acri@polimi.it (A. Acri), Eugene.Nijman@v2c2.at (E. Nijman), michael.klanner@tugraz.at (M. Klanner), Guenter.Offner@avl.com (G. Offner), roberto.corradi@polimi.it (R. Corradi). 
Specific methods within FEM and BEM framework were developed with the aim to analyze and rank different radiating surfaces of a body, such as panel contribution analysis (PCA) [6-8]. In addition to FEM and BEM, the wave based method (WBM) has been developed as an alter-native method for solving steady-state acoustic problems in the mid-frequency range [9]. It is based on an indirect Trefftz approach [10-12] and the field variables are expressed in terms of globally defined shape functions, which are the exact solutions of the homogeneous governing differential equation, but which do not necessarily satisfy the boundary conditions $[9,13]$. The wave models are substantially smaller than equivalent FEM and BEM counterparts and exhibit an increased computational efficiency $[14,15]$. The WBM is nowadays applied to model many different problems, for example interior acoustic problems [13,16-18], exterior acoustic problems [19-23], plate and membrane problems [24-28], coupled vibro-acoustic problems $[9,11,16]$ or poroelastic material modeling $[29,30]$. A wide overview on the WBM can be found in [31]. Within the WBM framework, also a surface con-tribution analysis (SCA) was proposed to assess and rank the different radiating surfaces of the investigated system [32]. In this paper the SCA is used to evaluate the noise contributions of the different radiating surfaces. SCA consists in the decomposition of the boundary conditions of the WBM model, assuming the linearity of the vibrational problem associated to the generation of the vibrations on the structure surface, for which the superposition principle is valid. In common industrial applications, the engine surface vibrations are derived from flexible multi-body dynamic (FMBD) simulations [33]. FMBD systems are non-linear systems that exhibit large rigid body motion with associated small flexible deformations of the bodies [34-36]. FMBD models of internal combustion engines may have inaccuracies, which are caused by parameter uncertainties: joint clearances, friction, lubrication, load estimation, material non-uniformities, manufacturing and assembly errors are examples of factors influencing the model uncertainties. These inaccuracies generate also variations in the acoustic response and in the assessment of the radiating surfaces.

In literature, several methods can be found to formally assess the effects of uncertainties in mechanical systems. The Monte Carlo (MC) approach is an extensively used method in dynamic models. It consists of repeated random sampling of a set of system parameters to obtain the uncertainty distribution via numerical simulation results [37-39]. Being computationally demanding, Latin Hypercube Sampling [40] and Bayesian methods [41,42] were introduced to overcome classical MC limitations. In this paper, a Monte Carlo methodology is applied to determine the influence of the variability of statistically independent excitations on numerical results of WBM for the computation of acoustic exterior radiation.

In particular the paper focuses on the influence of cylinder cyclic variability on the assessment of radiating surfaces via a SCA. Cyclic variability has been observed since the earliest scientific studies of internal combustion engines $[43,44]$. Although in the beginning the predominant understanding of cyclic variability was that cycle-to-cycle variations were of stochastic nature $[45,46]$, there have been many attempts to analyze the cycle-to-cycle variations by applying deterministic methods from nonlinear dynamical systems and chaos theory [4751]. In general, it can be said that the physiochemical processes in an internal combustion engine are influenced by many factors like composition of fuel air mixtures, amount of recycled gases in the combustion chamber, engine aereodynamics or engine operating conditions. In this paper the cylinder pressure experimentally obtained from engine on a test bed and the cylinder pressure variability is sta-tistically treated and used as the input for the multi-body dynamic si-mulations. Surface normal velocities obtained from the FMBD simula-tions are used as boundary conditions for the WBM on which SCA is applied.

\section{Wave based method for exterior radiation problems}

\subsection{Problem definition}

Let us consider an exterior acoustic problem with a vibrating structure which boundary $\partial \Omega$ is surrounded by an unbounded 3-dimensional fluid domain $\Omega$. The fluid is characterized by its speed of sound $c$ and density $\rho$. Assuming that the system is linear, the fluid inviscid and isotropic and that the process is adiabatic, the steady state acoustic pressure field is governed by the homogeneous Helmholtz Eq. [52]

$\nabla^{2} p(\boldsymbol{r})+k^{2} p(\boldsymbol{r})=0$

where $\nabla^{2}=\frac{\partial^{2}}{\partial x^{2}}+\frac{\partial^{2}}{\partial y^{2}}+\frac{\partial^{2}}{\partial z^{2}}$ is the Laplace operator, $p$ the acoustic pressure, $\boldsymbol{r}$ the position vector, $k=\frac{\omega}{c}$ is the wave number (the ratio between frequency $\omega$ and speed of sound $c$ ). In Eq. (1) the source term is set to zero. There are three possible boundary conditions associated to this problem, assuming that the solid and the fluid structures are not coupled:

- Imposed normal velocity (Neumann boundary condition)

$$
v_{n}(\boldsymbol{r})=\frac{\mathrm{i}}{\rho \omega} \frac{\partial p(\boldsymbol{r})}{\partial \boldsymbol{n}}=\bar{v}_{n}(\boldsymbol{r}), \quad \forall \boldsymbol{r} \in \partial \Omega_{v}
$$

- Imposed acoustic pressure (Dirichlet boundary condition)

$$
p(\boldsymbol{r})=\bar{p}(\boldsymbol{r}), \quad \forall \boldsymbol{r} \in \partial \Omega_{p}
$$

- Imposed normal impedance (Mixed boundary conditions)

$$
p(\boldsymbol{r})=\bar{Z}_{n}(\boldsymbol{r}) v_{n}(\boldsymbol{r})=\bar{Z}_{n}(\boldsymbol{r}) \frac{\mathrm{i}}{\rho \omega} \frac{\partial p(\boldsymbol{r})}{\partial \boldsymbol{n}}, \quad \forall \boldsymbol{r} \in \partial \Omega_{z}
$$

where $i$ is the imaginary unit, $\boldsymbol{n}$ is the normal vector and $\bar{Z}_{n}$ denotes the normal impedance. Furthermore, $\partial \Omega_{p} \cup \partial \Omega_{v} \cup \partial \Omega_{z}=\partial \Omega$. Considering an exterior radiation problem, where the acoustic waves are radiating towards infinity, the Sommerfeld radiation condition is applied on the boundary, $\Gamma_{\infty}$ in order to ensure that no acoustic energy is reflected at infinity, see [53]:

$\lim _{|\boldsymbol{r}| \rightarrow \infty}\left(|\boldsymbol{r}|\left(\frac{\partial p(\boldsymbol{r})}{\partial|\boldsymbol{r}|}+\mathrm{i} k p(\boldsymbol{r})\right)\right)=0$

$\Gamma_{\infty}$ is the boundary placed in the far field and represents the limit of the fluid domain in a numerical model (see Fig. 1a). As described in [21] and [54], the WBM includes an additional treatment to tackle exterior radiation problems with the introduction of an artificial truncation boundary that defines two sub-domains: a bounded part and an unbounded part. In the bounded region of the domain, the WBM formulation for interior problems is applied, whereas in the unbounded region, radiating functions, which satisfy the Sommerfeld radiation condition, are employed. The modeling strategy is shown in Fig. 1b, where an internal combustion engine is investigated.

\subsection{Bounded region}

In general, the WBM requires a convex domain to ensure convergence towards the exact solution [9,13] (see [55] for cases, where the WBM can be directly applied to non-convex domains). Therefore, it is necessary to decompose non-convex domains into a set of $N_{v}$ convex 


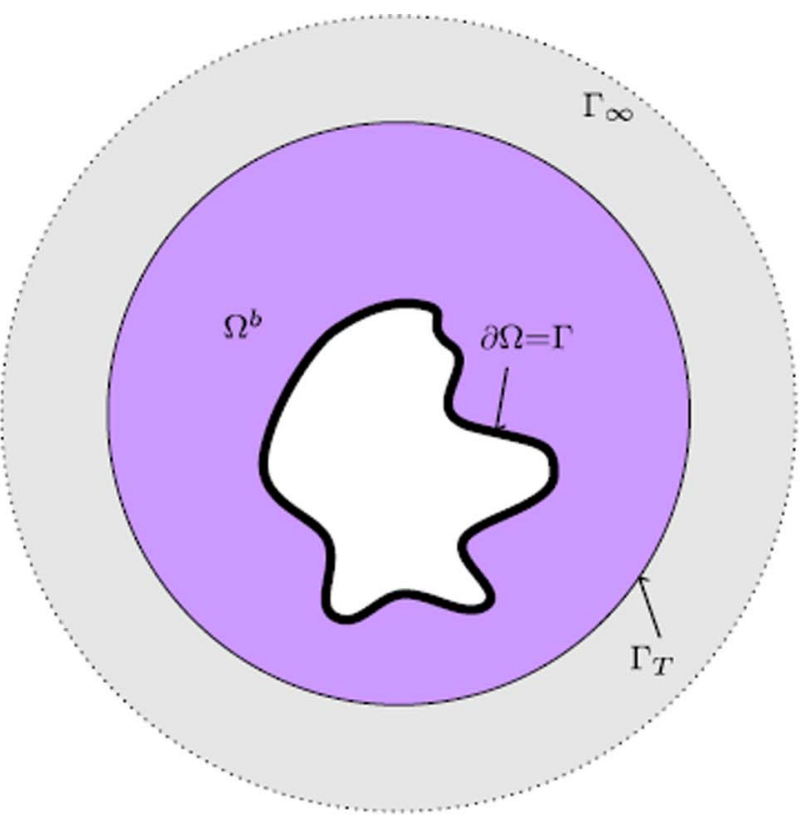

(a)

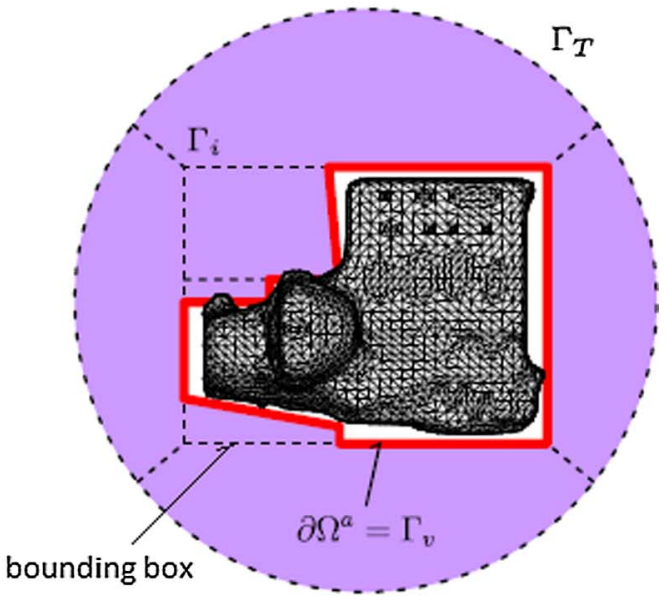

(b)

Fig. 1. Application of WBM for one-way coupled structural-acoustic unbounded problems: (a) the concept of a truncation boundary $\Gamma_{T}$ and (b) the modeling strategy [54].

sub-domains $\Omega=\bigcup_{\alpha=1}^{N_{v}} \Omega^{(\alpha)}$ and enforce appropriate continuity conditions between the sub-domains. Within one sub-domain $\Omega^{(\alpha)}$, the exact acoustic pressure solution is approximated with a linear combination of $n_{b}^{(\alpha)}$ acoustic wave functions $\boldsymbol{\Phi}_{B, j}^{(\alpha)}$

$p^{(\alpha)}(\boldsymbol{r}) \approx \sum_{j=1}^{n_{b}^{(\alpha)}} \mathbf{p}_{B, j}^{(\alpha)} \boldsymbol{\Phi}_{B, j}^{(\alpha)}(\boldsymbol{r})+\bar{p}_{q}^{(\alpha)}=\boldsymbol{\Phi}_{B}^{(\alpha)} \mathbf{p}_{B}^{(\alpha)}+\bar{p}_{q}^{(\alpha)}$

where the unknown weighting factors, gathered in the vector $\mathbf{p}_{B}^{(\alpha)}$, are the degrees of freedom of the problem and $\bar{p}_{q}^{(\alpha)}$ is the source term (the particular solution of the Helmholtz equation). As discussed in [13], the acoustic wave functions are defined by

$\boldsymbol{\Phi}_{B, j}^{(\alpha)}(\boldsymbol{r})=\left\{\begin{array}{l}\cos \left(k_{x B, r}^{(\alpha)} x\right) \cos \left(k_{y B, r}^{(\alpha)} y\right) \mathrm{e}^{-\mathrm{i} k_{z B, r}^{(\alpha)} z} \\ \cos \left(k_{x B, s}^{(\alpha)} x\right) \mathrm{e}^{-\mathrm{i} k_{y B, s}^{(\alpha)} y} \cos \left(k_{z B, s}^{(\alpha)} z\right) \\ \mathrm{e}^{-\mathrm{i} k_{x B, t}^{(\alpha)} x} \cos \left(k_{y B, t}^{(\alpha)} y\right) \cos \left(k_{z B, t}^{(\alpha)} z\right)\end{array}\right.$

The only condition needed to satisfy the Helmholtz equation is that the wave numbers in the different directions are related by the expression: $\left(k_{x B}^{(\alpha)}\right)^{2}+\left(k_{y B}^{(\alpha)}\right)^{2}+\left(k_{z B}^{(\alpha)}\right)^{2}=k^{2}$. In [9], it is suggested to consider the following wave number components

$\left(k_{x B, r}^{(\alpha)}, k_{y B, r}^{(\alpha)}, k_{z B, r}^{(\alpha)}\right)=\left[\frac{b_{1}^{(\alpha)} \pi}{L_{x}^{(\alpha)}}, \frac{b_{2}^{(\alpha)} \pi}{L_{y}^{(\alpha)}}, \pm \sqrt{k^{2}-\left(\frac{b_{1}^{(\alpha)} \pi}{L_{x}^{(\alpha)}}\right)^{2}-\left(\frac{b_{z}^{(\alpha)} \pi}{L_{y}^{(\alpha)}}\right)^{2}}\right]$
$\left(k_{x B, s}^{(\alpha)}, k_{y B, s}^{(\alpha)}, k_{z B, s}^{(\alpha)}\right)=\left[\frac{b_{3}^{(\alpha)} \pi}{L_{x}^{(\alpha)}}, \pm \sqrt{k^{2}-\left(\frac{b_{3}^{(\alpha)} \pi}{L_{x}^{(\alpha)}}\right)^{2}-\left(\frac{b_{4}^{(\alpha)} \pi}{L_{z}^{(\alpha)}}\right)^{2}}, \frac{b_{4}^{(\alpha)} \pi}{L_{z}^{(\alpha)}}\right]$
$\left(k_{x B, t}^{(\alpha)}, k_{y B, t}^{(\alpha)}, k_{z B, t}^{(\alpha)}\right)=\left[ \pm \sqrt{k^{2}-\left(\frac{b_{5}^{(\alpha)} \pi}{L_{y}^{(\alpha)}}\right)^{2}-\left(\frac{b_{6}^{(\alpha)} \pi}{L_{z}^{(\alpha)}}\right)^{2}}, \frac{b_{5}^{(\alpha)} \pi}{L_{y}^{(\alpha)}}, \frac{b_{6}^{(\alpha)} \pi}{L_{z}^{(\alpha)}}\right]$

with $b_{1}^{(\alpha)}, b_{2}^{(\alpha)}, b_{3}^{(\alpha)}, b_{4}^{(\alpha)}, b_{5}^{(\alpha)}$ and $b_{6}^{(\alpha)}=0,1,2, \ldots$ and $L_{x}^{(\alpha)}, L_{y}^{(\alpha)}$ and $L_{z}^{(\alpha)}$ are the smallest dimensions of the rectangular bounding box enclosing the sub-domain $\Omega^{(\alpha)}$.
In the WBM a weighted residual formulation is applied to minimize the boundary and interface errors and to determine $\boldsymbol{C}$ the unknown contribution factors $\mathbf{p}_{B}^{(\alpha)} \quad[9,13]$. Expanding the weighting functions with the same function sets as the pressure field (Galerkin approach) and minimizing the error for each sub-domain $\Omega^{(\alpha)}$, yields

$\left(\boldsymbol{p}_{\boldsymbol{w}}^{(\alpha)}\right)^{T}\left(\boldsymbol{A}^{(\alpha)} \boldsymbol{p}_{\boldsymbol{B}}^{(\alpha)}-\boldsymbol{f}^{(\alpha)}+\sum_{\beta=1, \beta \neq \alpha}^{N_{v}}\left[\boldsymbol{C}^{(\alpha, \beta)} \boldsymbol{p}_{\boldsymbol{B}}^{(\beta)}-\boldsymbol{f}^{(\alpha, \beta)}\right]\right)=0$

where $\mathrm{T}$ is the transpose, $\alpha$ and $\beta$ denote indices associated to the subdomains, $\boldsymbol{p}_{\boldsymbol{w}}^{(\alpha)}$ denotes the vector of weighting functions factors, $\boldsymbol{A}$ and are matrices resulting from the weighted residual formulation, $\boldsymbol{f}$ are vectors of known terms which depend on boundary conditions and the wave functions. The definitions of the matrices $\boldsymbol{A}$ and $\boldsymbol{C}$ and the vectors $\boldsymbol{f}$ can be found in [13]. The weighted residual formulation in Eq. (9) should hold for any combination of weighting function, which yields a set of linear equations where the unknown terms are the wave function contribution factors. The assemble of all sub-domains reads in matrix form:

$\left[\begin{array}{ccccc}\boldsymbol{A}^{(1)} & \boldsymbol{C}^{(1,2)} & \boldsymbol{C}^{(1,3)} & \ldots & \boldsymbol{C}^{\left(1, \boldsymbol{N}_{\boldsymbol{v}}\right)} \\ \boldsymbol{C}^{(2,1)} & \boldsymbol{A}^{(2)} & \boldsymbol{C}^{(2,3)} & \ldots & \boldsymbol{C}^{\left(2, \boldsymbol{N}_{\boldsymbol{v}}\right)} \\ \vdots & \vdots & \vdots & \ddots & \vdots \\ \boldsymbol{C}^{\left(\boldsymbol{N}_{\boldsymbol{v}}, 1\right)} & \boldsymbol{C}^{\left(\boldsymbol{N}_{\boldsymbol{v}}, 2\right)} & \boldsymbol{C}^{\left(\boldsymbol{N}_{\boldsymbol{v}}, 3\right)} & \ldots & \boldsymbol{A}^{\left(\boldsymbol{N}_{\boldsymbol{v}}\right)}\end{array}\right]\left[\begin{array}{c}\boldsymbol{p}_{\boldsymbol{B}}^{(1)} \\ \boldsymbol{p}_{\boldsymbol{B}}^{(2)} \\ \boldsymbol{p}_{\boldsymbol{B}}^{(3)} \\ \vdots \\ \boldsymbol{p}_{\boldsymbol{B}}^{\left(N_{\boldsymbol{v}}\right)}\end{array}\right]=\left[\begin{array}{c}\boldsymbol{b}^{(1)} \\ \boldsymbol{b}^{(2)} \\ \boldsymbol{b}^{(3)} \\ \vdots \\ \boldsymbol{b}^{\left(\boldsymbol{N}_{\boldsymbol{v}}\right)}\end{array}\right]$

with

$\boldsymbol{b}^{(\alpha)}=\boldsymbol{f}^{(\alpha)}+\sum_{\beta=1, \beta \neq \alpha}^{N_{v}} \boldsymbol{f}^{(\alpha, \beta)}$

The WBM matrix equation can also be expressed in a condensed form as

$\boldsymbol{A} \boldsymbol{p}_{B}=\boldsymbol{b}$ 


\subsection{Unbounded region}

In the unbounded domain, the solution has to fulfill both Sommerfeld radiation condition and Helmholtz equation. The unbounded subdomain is always concave, since it is defined by the unbounded region exterior to a sphere. However, the convexity requirement is only necessary for bounded sub-domains [56]. A valid basis set for the wave function expansions is given in [21,57] and in a spherical coordinate reference system it re $\boldsymbol{f}^{(\alpha, \beta)}$ ads

$p(r, \vartheta, \varphi)=\sum_{l=0}^{N_{U}} \sum_{m=-l}^{l} p_{l}^{m} h_{l}(k r) Y_{l m}(\theta, \varphi)+\bar{p}_{q}$

where $p_{l}^{m}$ are the Legendre polynomials, $Y_{l m}(\vartheta, \varphi)$ denote the spherical harmonics and $h_{l}(k r)$ are the spherical Hankel functions.

The modeling of exterior acoustic problems in WBM is done using an artificial spherical truncation boundary surface, as described in $[21,58]$. A bounding box, whose characteristic dimensions are the ones of the investigated radiating object, is introduced together with 6 elements between the truncation boundary $\Gamma_{T}$ and the bounding box, as shown in Fig. 1b.

\section{Surface contribution analysis}

\subsection{Application of boundary conditions to a wave based model}

In Eq. (11), the terms $\boldsymbol{b}^{(\alpha)}$ that constitute the known terms of the WB system equation are a sum of different $(\alpha \neq \beta)$ terms and $\boldsymbol{f}^{(\alpha)}$. Their expression is related to the boundary conditions described in Eqs. (2-4) and the coupling conditions between sub-domains. The vector $\boldsymbol{f}^{(\alpha)}$, which is only associated with the domain $\alpha$, can be written as

$\boldsymbol{f}^{(\alpha)}=\boldsymbol{f}_{v}^{(\alpha)}+\boldsymbol{f}_{p}^{(\alpha)}+\boldsymbol{f}_{Z}^{(\alpha)}+\boldsymbol{f}_{I v}^{(\alpha)}+\boldsymbol{f}_{I p}^{(\alpha)}$

The term associated to velocity boundary conditions is

$\boldsymbol{f}_{v}^{(\alpha)}=\int_{\partial \Omega_{\nu}^{(\alpha)}}\left(\boldsymbol{\Phi}_{B}^{(\alpha)}\right)^{T}\left(\bar{v}_{n}-\frac{\mathrm{i}}{\rho_{0} \omega}\left(\boldsymbol{n}^{(\alpha)}\right)^{T} \nabla \bar{p}_{q}^{(\alpha)}\right) d \Gamma$

The term associated to acoustic pressure boundary condition is

$\boldsymbol{f}_{p}^{(\alpha)}=\int_{\partial \Omega_{p}^{(\alpha)}} \frac{\mathrm{i}}{\rho_{0} \omega}\left(\nabla \boldsymbol{\Phi}_{B}^{(\alpha)}\right)^{\mathrm{T}} \boldsymbol{n}^{(\alpha)}\left(\bar{p}_{q}^{(\alpha)}-\bar{p}\right) d \Gamma$

The term associated to impedance boundary condition is

$\boldsymbol{f}_{Z}^{(\alpha)}=\int_{\partial \Omega_{Z}^{(\alpha)}}\left(\boldsymbol{\Phi}_{B}^{(\alpha)}\right)^{T}\left(\frac{\bar{p}_{q}^{(\alpha)}}{\bar{Z}_{n}}-\frac{\mathrm{i}}{\rho_{0} \omega}\left(\boldsymbol{n}^{(\alpha)}\right)^{T} \nabla \bar{p}_{q}^{(\alpha)}\right) d \Gamma$

The term associated to velocity continuity at interfaces with other subdomains is

$\boldsymbol{f}_{I v}^{(\alpha)}=\int_{\partial \Omega_{I V}^{(\alpha)}}\left(\boldsymbol{\Phi}_{B}^{(\alpha)}\right)^{T}\left(\frac{\mathrm{i}}{\rho_{0} \omega}\left(\boldsymbol{n}^{(\alpha)}\right)^{T} \nabla \bar{p}_{q}^{(\alpha)}\right) d \Gamma$

The term associated to acoustic pressure continuity at interfaces with other sub-domains is

$\boldsymbol{f}_{I p}^{(\alpha)}=\int_{\partial \Omega_{I p}^{(\alpha)}} \frac{\mathrm{i}}{\rho_{0} \omega}\left(\nabla \boldsymbol{\Phi}_{B}^{(\alpha)}\right)^{\mathrm{T}} \boldsymbol{n}^{(\alpha)}\left(\bar{p}_{q}^{(\alpha)}\right) d \Gamma$

The vectors $\boldsymbol{f}^{(\alpha, \beta)}$ resulting from the coupling are given by

$$
\begin{aligned}
\boldsymbol{f}^{(\alpha, \beta)}= & -\int_{\partial \Omega_{I v}^{(\alpha, \beta)}}\left(\boldsymbol{\Phi}_{B}^{(\alpha)}\right)^{T}\left(\frac{\mathrm{i}}{\rho_{0} \omega}\left(\boldsymbol{n}^{(\beta)}\right)^{T} \nabla \bar{p}_{q}^{(\beta)}\right) d \Gamma \\
& -\int_{\partial \Omega_{I p}^{(\alpha, \beta)}} \frac{\mathrm{i}}{\rho_{0} \omega}\left(\nabla \boldsymbol{\Phi}_{B}^{(\alpha)}\right)^{\mathrm{T}}\left(\boldsymbol{n}^{(\beta)}\right)^{T}\left(\bar{p}_{q}^{(\beta)}\right) d \Gamma
\end{aligned}
$$

where $\bar{v}_{n}$ and $\bar{p}$ are respectively the imposed velocity and acoustic pressure at the boundary, $\bar{Z}_{n}$ is the imposed impedance at the boundary, $\bar{p}_{q}^{(\alpha)}$ is the particular solution of the Helmholtz equation (source term) in sub-domain $\alpha$ while $\bar{p}_{q}^{(\beta)}$ is the solution of sub-domain $\beta, \partial \Omega_{I}^{(\alpha)}$ is the coupling boundary with other sub-domains and $\partial \Omega_{I}^{(\alpha, \beta)}$ is the surface boundary that sub-domain $\alpha$ and $\beta$ share. Note that

$\partial \Omega_{I}^{(\alpha)}=\bigcup_{\beta=1, \beta \neq \alpha}^{N_{v}} \partial \Omega_{I}^{(\alpha, \beta)}$

and also

$\partial \Omega^{(\alpha)}=\partial \Omega_{v}^{(\alpha)} \cup \partial \Omega_{p}^{(\alpha)} \cup \partial \Omega_{Z}^{(\alpha)} \cup \partial \Omega_{I}^{(\alpha)}$

In exterior radiation problems, the boundary conditions of the bounded region are the ones in Eq. (14) and are introduced to solve the system in Eq. (12).

When complex geometries are investigated (e.g. internal combustion engines), boundary conditions such as structural velocities can be mapped on WB sub-domains adopting an MLV (multi local velocity) algorithm as described in [54].

\subsection{Problem formulation for a wave based surface contribution analysis}

The purpose of SCA is to separately calculate the solution of Eq. (12) decomposing the area on which the boundary conditions are calculated. For the sake of simplicity, in the following expressions the source terms $\bar{p}_{q}^{(\alpha)}$ are set equal to zero, although, for a more general source contribution analysis formulation, it is possible to consider also these terms. Neglecting the source terms, it is straight forward to analyze separately the noise radiated from the different surfaces. The terms $\boldsymbol{b}^{(\alpha)}$ in Eq. (10) are defined as sum of different boundary conditions, which are expressed in Eqs. (15) and (16) and are applied on different surfaces, $\partial \Omega_{v}^{(\alpha)}, \partial \Omega_{p}^{(\alpha)}, \partial \Omega_{Z}^{(\alpha)}$ and $\partial \Omega_{I}^{(\alpha)}$.

Consider a boundary surface selection $\partial \Omega_{j}$ such that $\partial \Omega=\bigcup_{j=1}^{N s} \partial \Omega_{j}$, being $N s$ the total number of surface selections. Introducing Eq. (17) and Eq. (18) in Eq. (11), the boundary conditions associated to $\partial \Omega_{j}$ can be expressed as

$\boldsymbol{b}_{\boldsymbol{j}}=\left[\begin{array}{c}\boldsymbol{b}_{\boldsymbol{j}}^{(1)} \\ \boldsymbol{b}_{j}^{(2)} \\ \boldsymbol{b}_{j}^{(3)} \\ \vdots \\ \boldsymbol{b}_{\boldsymbol{j}}^{\left(\boldsymbol{N}_{\boldsymbol{v}}\right)}\end{array}\right]=\left[\begin{array}{c}\boldsymbol{f}_{j}^{(1)}+\sum_{\beta=1, \beta \neq 1}^{N_{v}} \boldsymbol{f}_{j}^{(1, \beta)} \\ \boldsymbol{f}_{j}^{(2)}+\sum_{\beta=1, \beta \neq 2}^{N_{v}} \boldsymbol{f}_{j}^{(2, \beta)} \\ \boldsymbol{f}_{j}^{(3)}+\sum_{\beta=1, \beta \neq 3}^{N_{v}} \boldsymbol{f}_{j}^{(3, \beta)} \\ \vdots \\ \boldsymbol{f}_{j}^{\left(N_{v}\right)}+\sum_{\beta=1, \beta \neq N_{v}}^{N_{v}} \boldsymbol{f}_{j}^{\left(N_{v}, \beta\right)}\end{array}\right]$

where $\boldsymbol{f}_{j}^{(\alpha)}$ and $\boldsymbol{f}_{j}^{(\alpha, \beta)}$ differ from Eqs. (15) and (16) only because of the different integration regions, $\partial \Omega_{v, j}^{(\alpha)} \partial \Omega_{p, j}^{(\alpha)}, \partial \Omega_{Z, j}^{(\alpha)}, \partial \Omega_{I v, j}^{(\alpha)}, \partial \Omega_{I p, j}^{(\alpha)}, \partial \Omega_{I v, j}^{(\alpha, \beta)}$ and $\partial \Omega_{I p, j}^{(\alpha, \beta)}$. Moreover, the relation $\partial \Omega_{x}^{(\alpha)}=\bigcup_{j=1}^{N s} \partial \Omega_{x, j}^{(\alpha)}$ holds for every integration surface. Given that, the WBM system of Eq. (12) can be solved considering the known terms $\boldsymbol{b}_{\boldsymbol{i}}$, it follows 


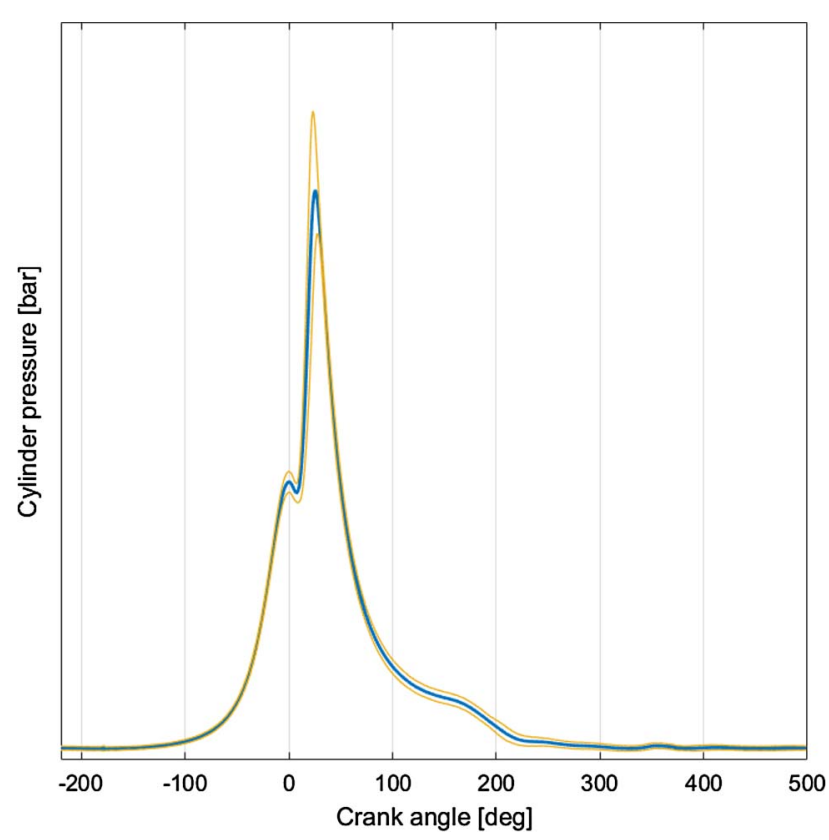

Fig. 2. Cyclic variability of measured cylinder pressure. In blue, the mean value. In orange, the $95 \%$ confidence interval of measured data. (For interpretation of the references to colour in this figure legend, the reader is referred to the web version of this article.)

$A p_{B j}=b_{j}$

The terms $\boldsymbol{p}_{\boldsymbol{B}}$, correspond to the contribution of surface $\partial \Omega_{j}$ to the global solution of the associated radiation problem. Since the WBM

formulation solves the Helmholtz Eq. (1), for which solution the superposition principle is valid, and considering the validity of Eqs. (17) and (18), also the solutions $\boldsymbol{p}_{B j}$, obtained considering the known terms $\boldsymbol{b}_{j}^{(\alpha)}$, can be linearly summed

$\boldsymbol{p}_{\boldsymbol{B}}=\sum_{j=1}^{N_{S}} \boldsymbol{p}_{\boldsymbol{B} \boldsymbol{j}}$

\section{Cyclic variability of cylinder pressure}

In this paper, the influence of cyclic variability on the results obtained from acoustic radiation WBM model is investigated. Particular attention is paid to the assessments of the different radiating surfaces of the engine. Therefore, the effects of cyclic variability are investigated using the SCA methodology described in Section 3. The cylinder pressure is given as an external excitation to the investigated FMBD system and then the structural vibration evaluated with FMBD simulation are used as boundary conditions for the WBM analysis. This pressure was measured on a GDI engine running under full load operating conditions with constant engine cycle averaged speed of $3000 \mathrm{rpm}$ and firing crank angle of $10 \mathrm{deg}$.

The measured data consists of 16 periods that are used as 16 sample cycles (one cycle correspond to two complete crankshaft rotations being a 4-stroke engine). In Fig. 2, the blue line shows the mean value of the cylinder pressure measured, while the orange lines correspond to the 95\% confidence interval of the measured data (two times the standard deviation). Due to confidentiality of measured data, values on y-axis of Fig. 2 are omitted. The maximum pressure measured exhibits a Gaussian distribution with a standard deviation of 8.6 bar. From these pressure data, an equivalent distributed force is derived, which is applied in normal direction both on piston heads and on the walls of the combustion chambers. Each time history is obtained by mixing the 16 measured cycles in random sequence. Each excitation in a cylinder has a time history which is independent from the excitations in the other cylinders and the statistical dispersion of the excitations correspond to the one shown in Figs. 2 and 3. Fig. 3 shows the statistical distribution of the resultant force applied on the piston. This distribution is obtained from a MC approach consisting of 30 simulations, according with [59]. The frequency spectra in Fig. 3 are input to the FMBD considering 3 consecutive engine periods (i.e. $0.12 \mathrm{~s}$ ) windowed with Hanning function, resulting in a frequency step of $8.3 \mathrm{~Hz}$. From Fig. 3, it can be seen that the frequency spectrum of the applied force is characterized by a series of tonal components. The frequencies of these tones depend on the engine speed. The first peak in the frequency spectra occurs at the 0.5th engine order, $25 \mathrm{~Hz}$, and the other harmonics at its multiples. Most of the energy is contained below $250 \mathrm{~Hz}$. Concerning the statis-tical distribution of data, it can be said that the standard deviation is relatively low in correspondence of the cylinder firing frequency $(25 \mathrm{~Hz}$ and multiples). The dispersion in the frequency spectra, is higher compared to the one in time domain. The main reason is that the higher frequency content is influenced by the shape and the smoothness of the excitation time history [60]. In fact, the variability of the cylinder pressure spectrum is not only influenced by the maximum pressure, but also (and especially) by the shape of the pressure time history, as confirmed in Fig. 4 where the first and second time derivative of the pressure time history are plotted.

\section{Influence of cyclic variability on the assessment of vibrating surfaces}

Several methodologies can help engineers to assess and rank radiating surfaces of a vibrating structures. Unfortunately, numerical models are deterministic, and do not consider system or excitation variability. The aim of this section is to introduce a methodology which helps to deal with uncertainties due to excitation variability. In this section, the influence of cyclic cylinder variability on the assessment and ranking of radiating engine surfaces is investigated.

With this purpose, the measured excitations shown in Section 4 are used to model the engine dynamics. Structural vibrations are derived via FMBD simulation. The structural vibration are used as boundary conditions of the acoustic problem and with a SCA radiating surfaces are finally ranked. Considering that excitations exhibit a certain variability, different FMBD simulations are performed in a MC scheme. The acoustic results are finally statistically treaded in order to under-stand how excitation variability influences the acoustic ranking and assessment.

\subsection{The investigated model}

The investigated system is a four-cylinder internal combustion engine running at a full load condition with constant engine cycle averaged speed of $3000 \mathrm{rpm}$ (although the methodology can be applied for any engine running condition). Fig. 5 shows the 3D model investigated. The engine block is the radiating body investigated in the SCA and consists of 1,53,728 Degrees of Freedom (DoFs). The material selected is steel with an elasticity modulus of $2,10,000 \mathrm{~N} / \mathrm{mm}^{2}$, a Poisson ration of 0.3 and a density of $8076 \mathrm{~kg} / \mathrm{m}^{3}$. The structural vibrations are evaluated with a FMBD in time domain using the software AVL EXCITE [58]. The only excitation considered in the FMBD simulation is the cylinder gas pressure. Frequency domain results are derived from time domain simulated data, considering 3 engine periods and adopting a Hanning window. Once frequency domain results are extracted from FMBD simulation, SCA is performed within WBM framework implemented in AVL EXCITE [58].

Regarding the FMBD simulation, the total amount of time needed 


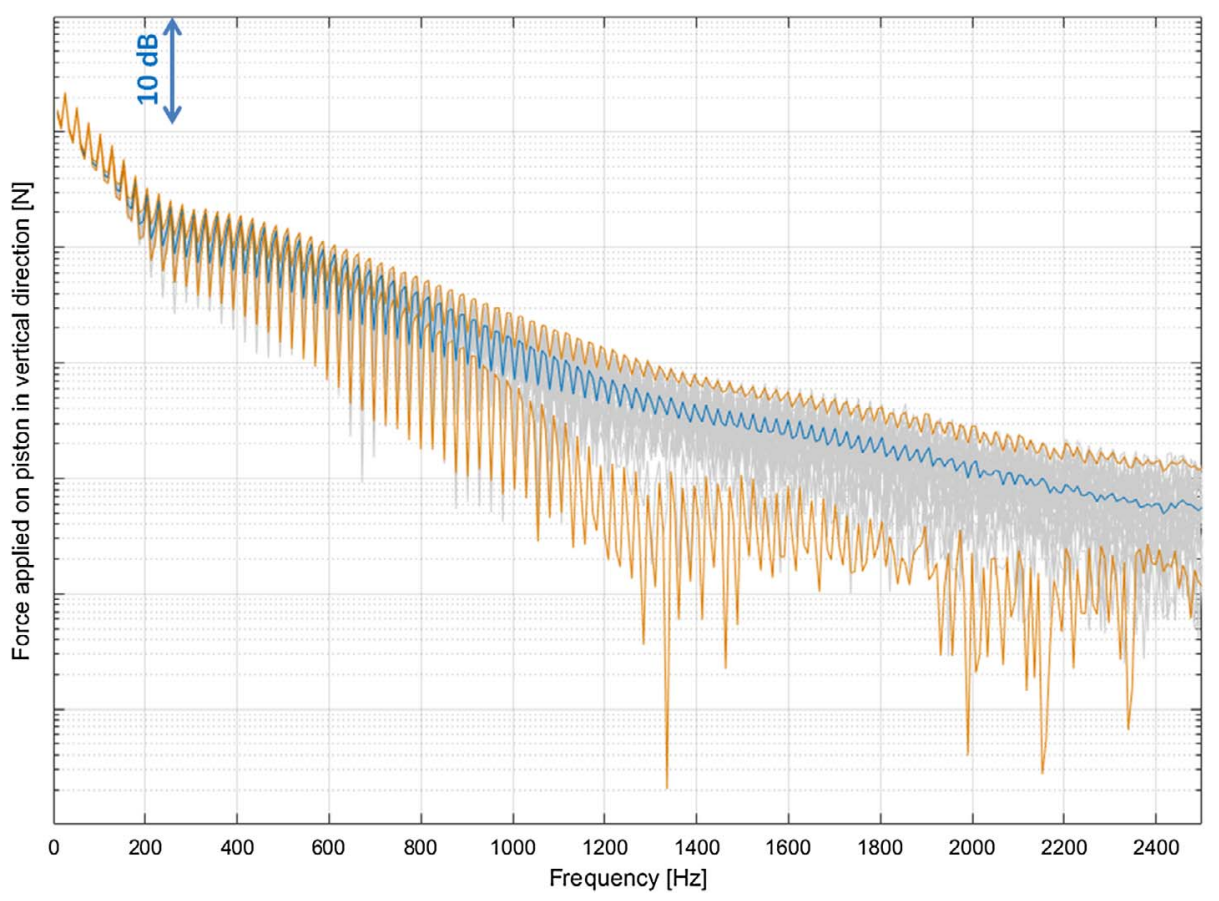

Fig. 3. Cyclic variability of piston force frequency spectra. In blue, the mean value. In orange, the $95 \%$ confidence interval of measured data. In grey, all the different samples of the MC approach. (For interpretation of the references to colour in this figure legend, the reader is referred to the web version of this article.)
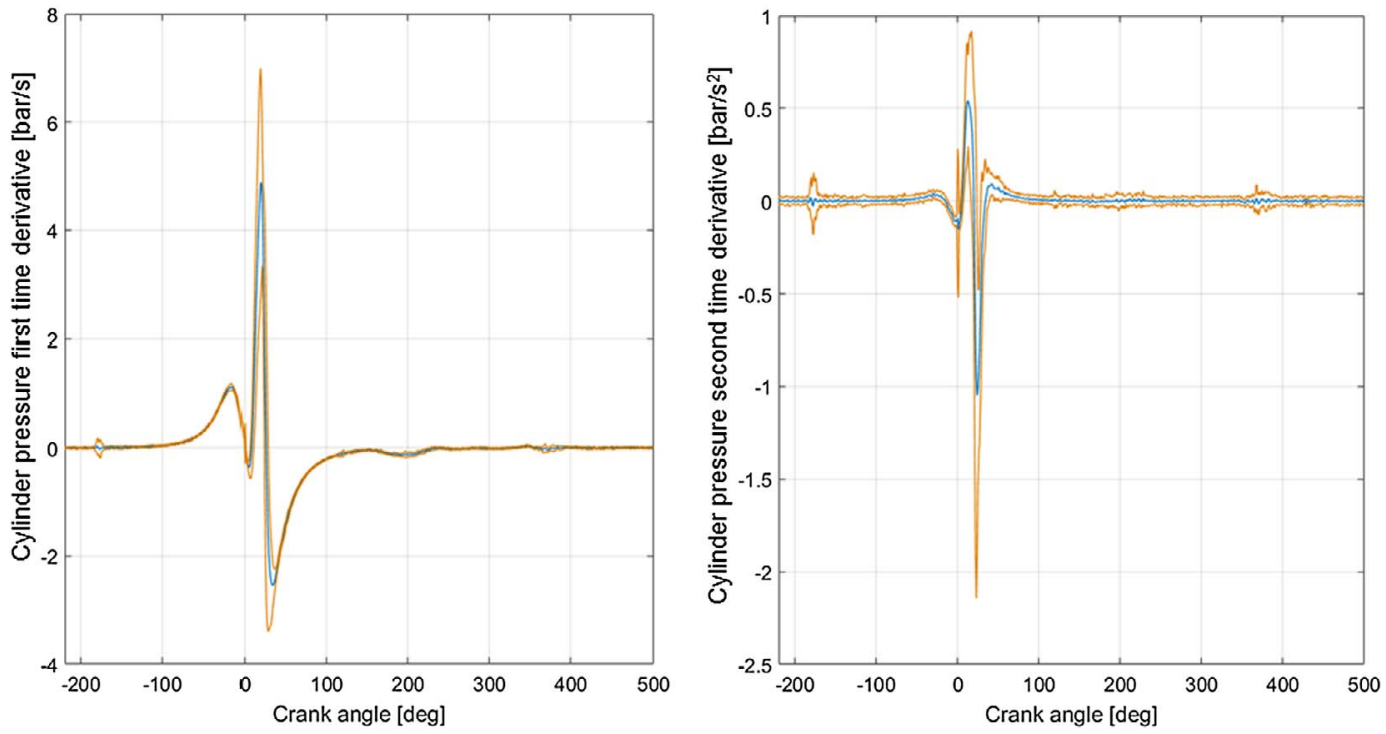

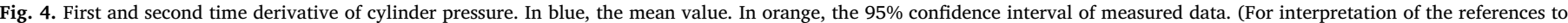
colour in this figure legend, the reader is referred to the web version of this article.)

for the computation is significantly influenced by the number of DoFs of the system. Hence, a condensed model with a reduced number of DoFs, equivalent to the full model [61-63], is considered for the simulation. The condensed number of DoFs of the engine block is 1666. All modes up to $3 \mathrm{kHz}$ are included into the reduced model. The dynamic system of equations is solved in time domain and the resulting data are transformed into frequency domain. Once the full multi-body simulations are performed in a Monte Carlo scheme, normal surface velocities are transformed into the frequency domain and are used as boundary condition in the SCA. This procedure will allow the evaluation of statistical information regarding the influence of cylinder cyclic variability on the assessment of radiating surfaces via a SCA.

Fig. 6 shows all the different surfaces investigated in the SCA. There are in total 27 different surfaces. Each surface is associated with an identification number as shown in Fig. 6: surfaces 1-4 are associated to the oil pan; 5 and 6 correspond to the air intake system; the cover head is from 7 to 9; the exhaust manifold in surface 10; 11 and 12 are placed in the front of the engine; the right hand side of the engine block consist of surfaces 13 and 14 while 15 and 16 belong the left hand side; the rear of the engine block is surface 17; 18 and 19 are the surfaces of the gearbox directly enclosing the differential; all the surfaces from 20 to 27 are associated with the gearbox.

\subsection{Influence of cyclic variability on noise radiation results}

The cyclic variability of the cylinder pressure influences the results 


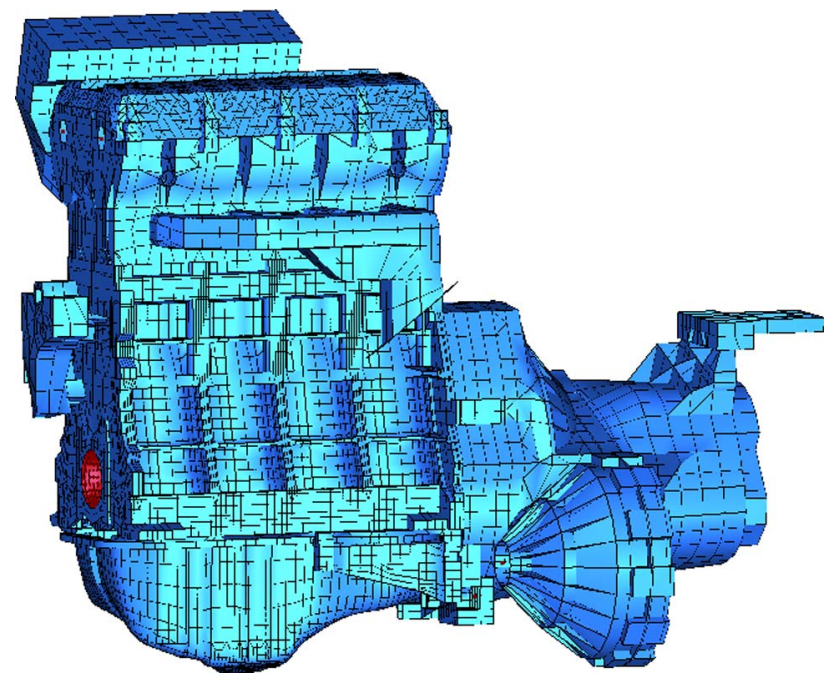

Fig. 5. The engine investigated.

of the multi-body dynamic simulation. Therefore, the structural normal velocities on the engine surface, which are the boundary conditions of the acoustic radiation model, may differ from one sample of the Monte Carlo methodology to another. In Fig. 7, the structural velocities on engine surface and the sound pressure level (SPL) on a spherical field point mesh of radius $2.2 \mathrm{~m}$ are shown for a frequency of $1450 \mathrm{~Hz}$. Fig. 7 compares results of 2 samples of the Monte Carlo algorithm, showing how relatively small changes on the structural side (on average $10 \mathrm{~dB}$ ) can lead to more than $30 \mathrm{~dB}$ of difference on the acoustic side, depending on the investigated field point node. Similar results were also obtained from experimental investigations [64]. These differences can also be caused by sound directivity variability.

Due to high variability of results and directivity oscillations, it was preferred to focus the attention on power quantities, which are associated to system surfaces. Fig. 8 shows the acoustic active output power evaluated on the spherical field point mesh placed in the far field having radius of $2.2 \mathrm{~m}$. The orange lines indicate the $95 \%$ confidence interval. The conclusion arising from Fig. 8 is that variability of results increases with increasing frequency, in agreement with the trend shown in Fig. 3.

\subsection{Influence of cyclic variability on surface contribution analysis}

Fig. 9 shows the average of acoustic radiated active power from all the different surfaces. Data are also compared with the overall power radiated from the whole engine (bottom line in Fig. 9). Results from SCA shown in Fig. 9 helps to rank the different surfaces in terms of emitted noise. E.g., at $1800 \mathrm{~Hz}$, where gear whine noise appears, sur-faces 20 and 23 (shown in Fig. 10) are the most relevant, with more than $10 \mathrm{~dB}$ of difference from all other surfaces. Fig. 10 also shows the normal surface velocities on the engine, highlighting how SCA helps to properly assess the radiating surfaces. In fact, although the oil pan and the bottom of the gearbox have similar velocity levels to the ones of surfaces 20 and 23 , their emitted acoustic power is negligible ( $10 \mathrm{~dB}$ difference or more).

The influence of cylinder cyclic variability on the assessment of the different radiating surfaces is shown in Figs. 11 and 12, where the acoustic radiated power at 600 and $1800 \mathrm{~Hz}$ is shown including the 95\% confidence interval bars (two times the standard deviation).

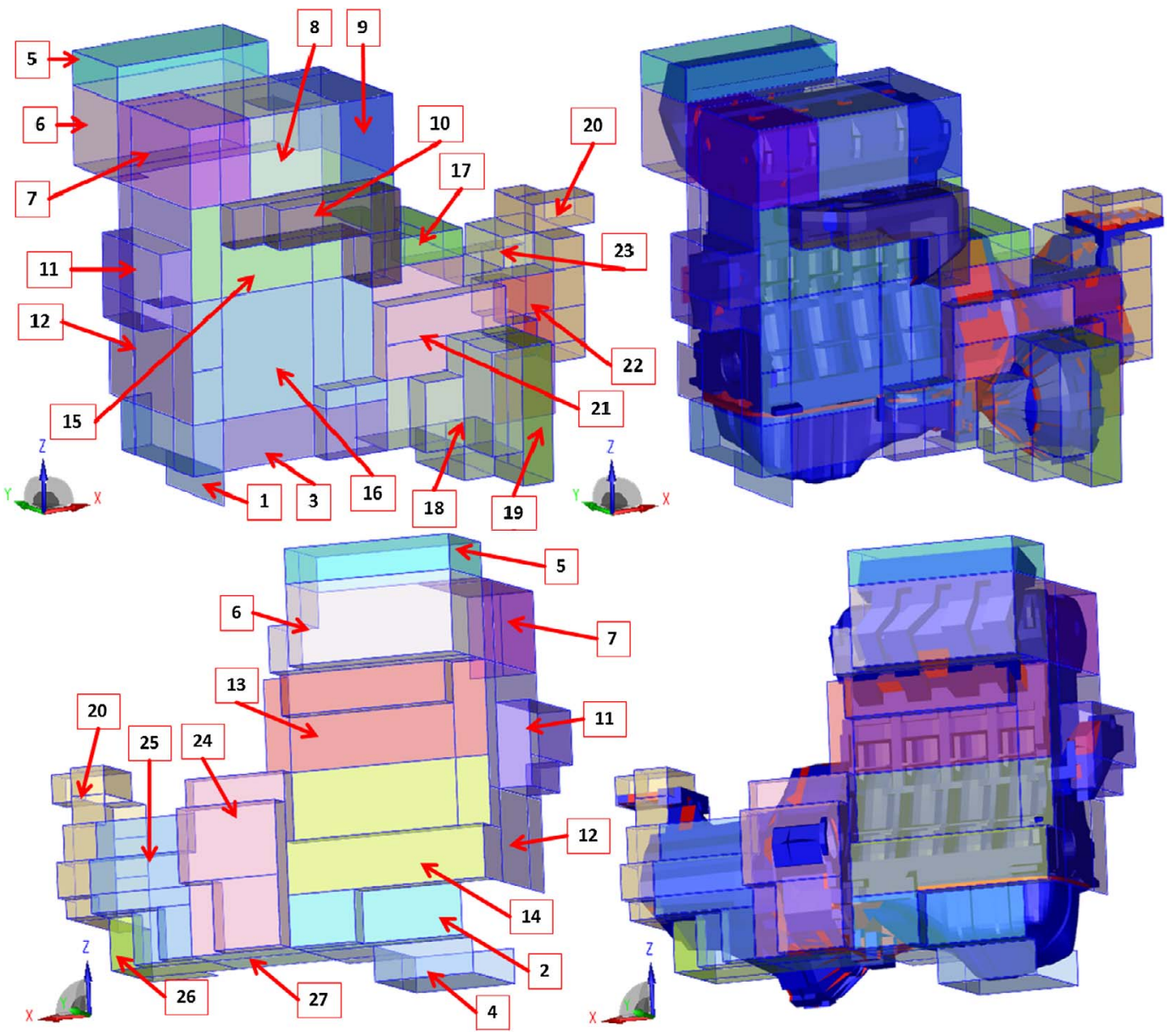

Fig. 6. The different surface selections investigated in the SCA. 
Normal velocity $[\mathrm{dB}]$
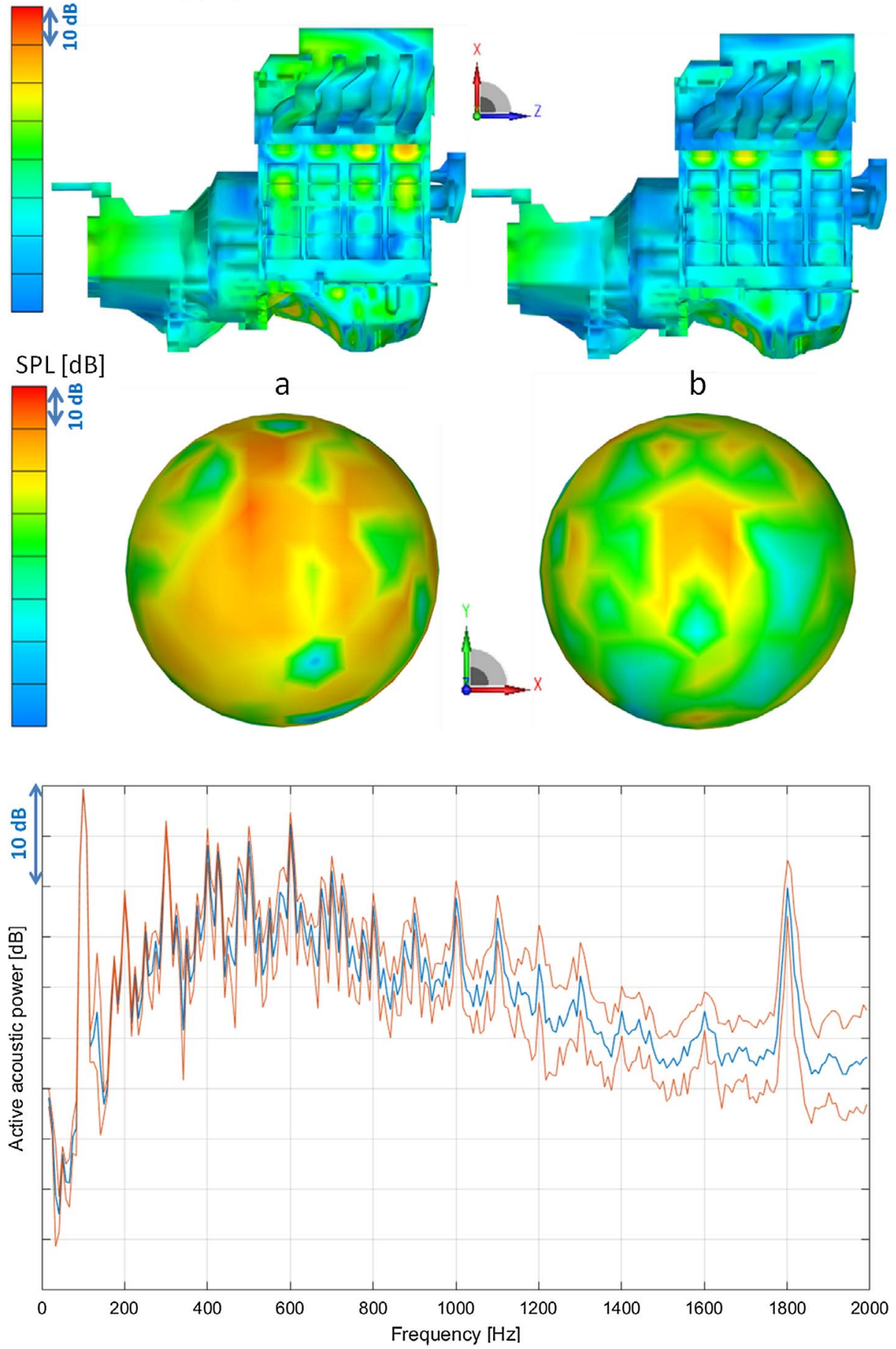

Fig. 7. Variability of results due to cyclic cylinder pressure variations at $1450 \mathrm{~Hz}$. Two different samples of the Monte Carlo calculations are shown (respectively a and b). Above, normal surface velocity. Below, SPL on a $2.2 \mathrm{~m}$ radius sphere.
Fig. 8. Active acoustic power evaluated on the spherical field point mesh placed in the far field having radius of $2.2 \mathrm{~m}$. In blue, the mean value. In red, the $95 \%$ confidence interval. (For interpretation of the references to colour in this figure legend, the reader is referred to the web version of this article.)
According to Fig. 8, also the results of the different surface selections are affected by variability which increases with frequency. Similar considerations may also arise from the analysis of other frequencies.

As shown in Figs. 11 and 12, with increasing variability of results, the $95 \%$ confidence interval bars of different surfaces may overlap. Therefore, it may happen that it is not possible to univocally rank the different panels. As for example in Fig. 12 at $1800 \mathrm{~Hz}$, it is not completely clear if the noise produced from surface 23 always has higher intensity than the one produced from surface 20.
To deal with this, the cross correlation between the sources is investigated. As an example, Fig. 13 shows the Pearson correlation coefficients $c c$ between the different power results at $1800 \mathrm{~Hz}$. The Pearson correlation coefficient is defined as the ratio between the covariance of two random variables, $A$ and $B$, and the product of their standard deviations, respectively $\sigma_{A}$ and $\sigma_{B}$, [65]

$c c=\frac{\operatorname{cov}(A, B)}{\sigma_{A} \sigma_{B}}$. 


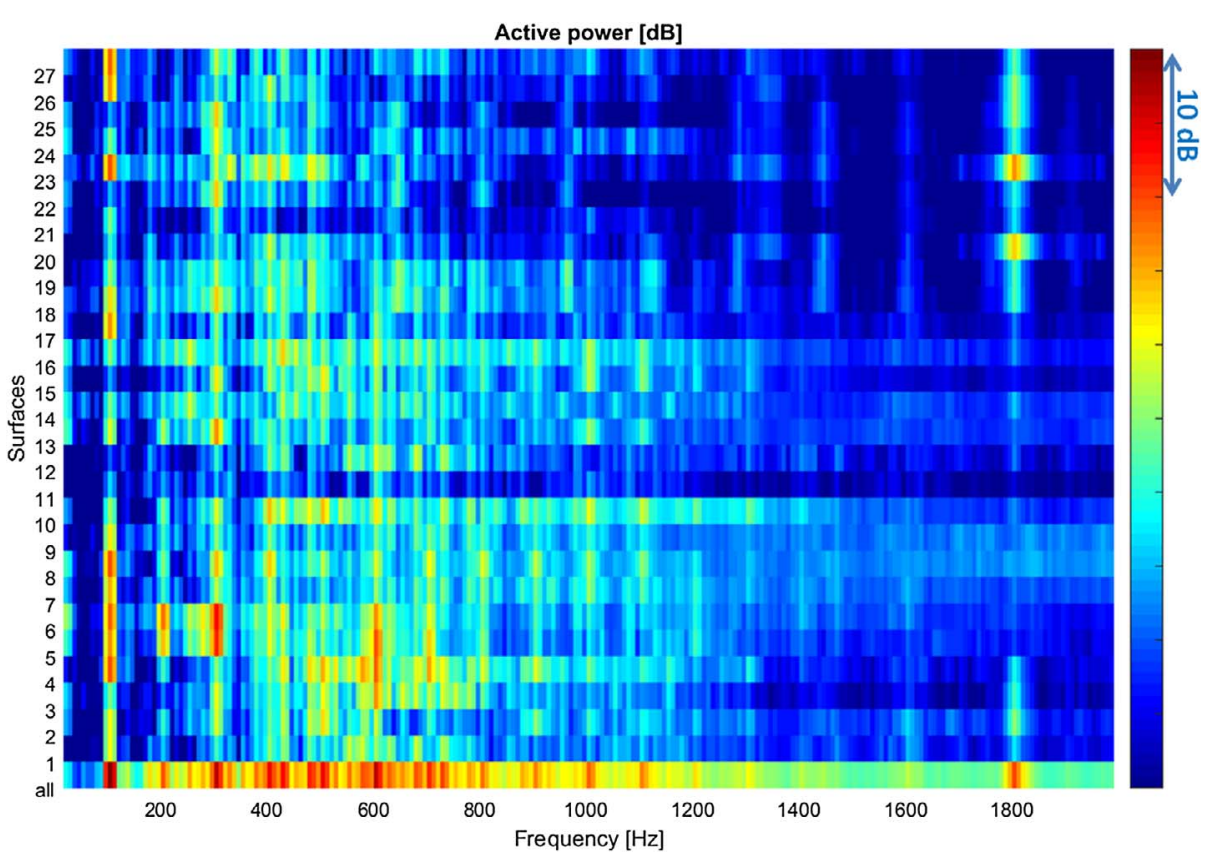

Normal velocity $[\mathrm{dB}]$
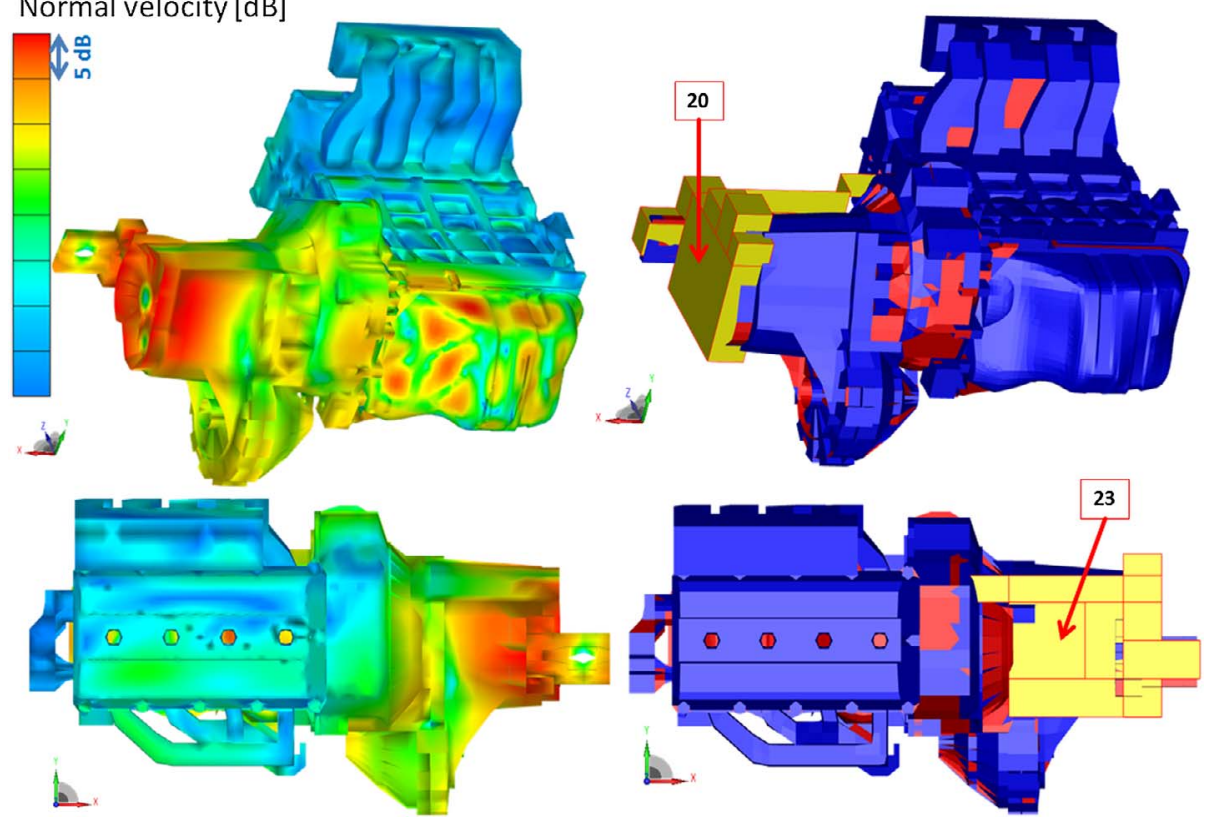

Fig. 9. Active acoustic power from the different surfaces. Mean values of the Monte Carlo method.
Fig. 10. Comparison between surface normal velocity and the position of the most radiating surfaces (in yellow) at $1800 \mathrm{~Hz}$. (For interpretation of the references to colour in this figure legend, the reader is referred to the web version of this article.)
From Fig. 13, it is clear that all the contributions to the overall noise from oil pan and gear box surfaces are strongly correlated to each other. Hence, it is possible to state that acoustic power radiated from surfaces 20 and 23 are correlated. Being correlated, the contribution of surface 23 is the most relevant at $1800 \mathrm{~Hz}$, because increasing contribution of surface 20 are correlated with increasing contribution of surface 23 .

Given two or more correlated contributions, one may intuitively think that they can be ranked using only mean values despite of high variability. To quantify how much the ranking based on mean values of radiated power is affected from system variability, the difference between projections is investigated. Considering the power radiated from two surfaces $A$ and $B$, respectively $P_{A}$ and $P_{B}$, their difference $\left(P_{A}-P_{B}\right)$ has a mean value $\mu_{A B}$ and a standard deviation $\sigma_{A B}$. The probability that
$P_{A}$ is grater that $P_{B}$ is proportional to the integral between zero and $+\infty$ of the Gaussian probability function generated using $\mu_{A B}$ and $\sigma_{A B}$. This information tell us that at $1800 \mathrm{~Hz}$, although the $95 \%$ confidence intervals of surfaces 20 and 23 overlap, surface 23 is always radiating more power (in accordance with their Pearson correlation coefficient shown in Fig. 13). This is not the case for surfaces 4 and 5 at $600 \mathrm{~Hz}$. Their Pearson correlation coefficient is 0.53 and only in the $58 \%$ of the cases surface 5 radiates more power than surface 4, as shown in Fig. 14. The elements in Fig. 14 indicate the probability that the contribution $i$ on the row is greater or equal to the contribution $j$ on the column. For the sake of simplicity, only the 4 most relevant radiating surfaces are considered. 
$600 \mathrm{~Hz}$

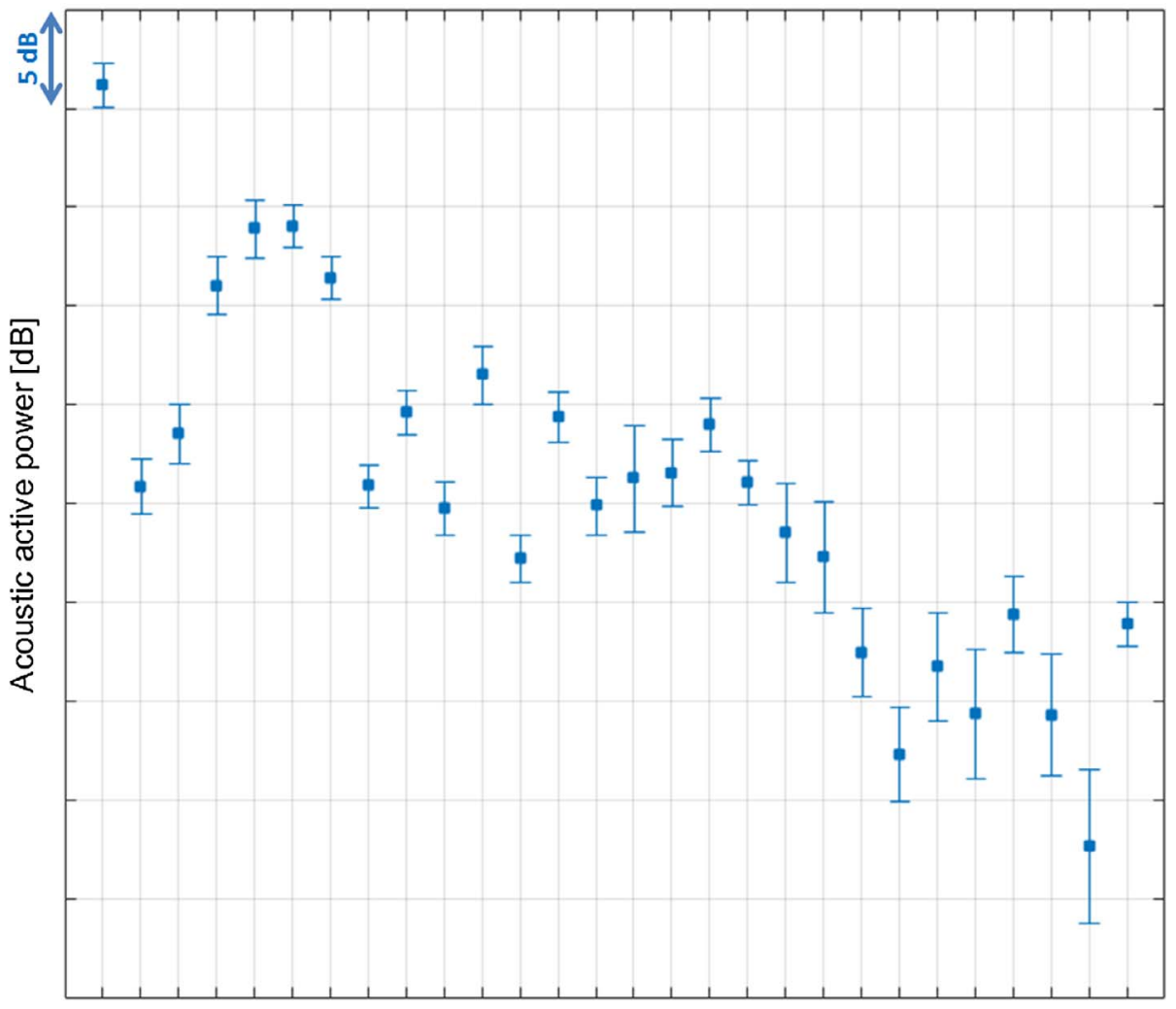

all $1 \quad 2 \quad 3 \quad 4 \quad 5 \quad 6 \quad 7 \quad 8 \quad 9101112131415161718192021222324252627$

Surfaces

$1800 \mathrm{~Hz}$

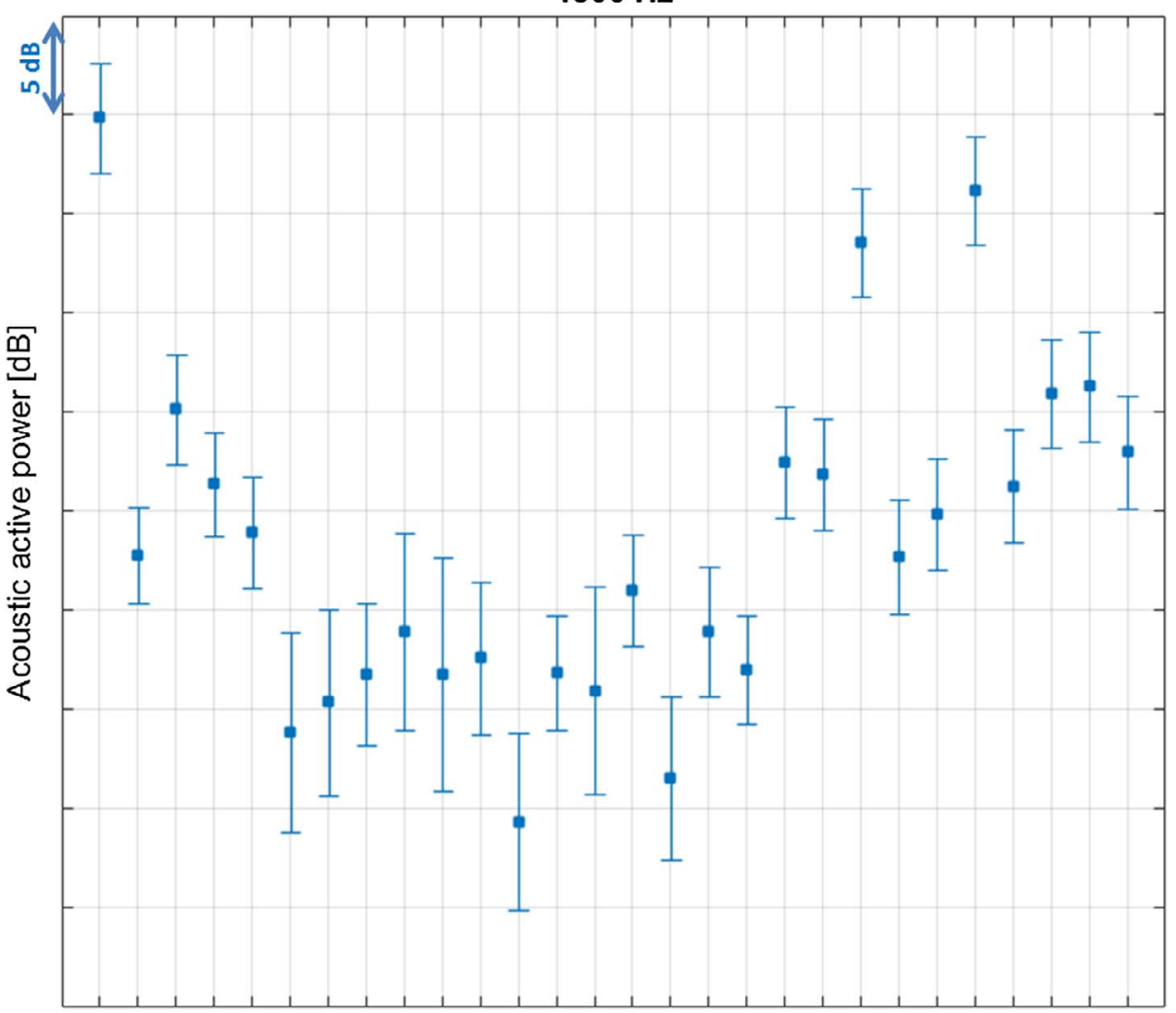

all $1 \begin{array}{lllllllllll}2 & 3 & 4 & 5 & 6 & 7 & 8 & 9 & 101112131415161718192021222324252627\end{array}$

Surfaces
Fig. 11. Acoustic active power of all different surfaces at $600 \mathrm{~Hz}$. The bars consider the $95 \%$ envelop of results.
Fig. 12. Acoustic active power of all different surfaces at $1800 \mathrm{~Hz}$. The bars consider the $95 \%$ envelop of results. 


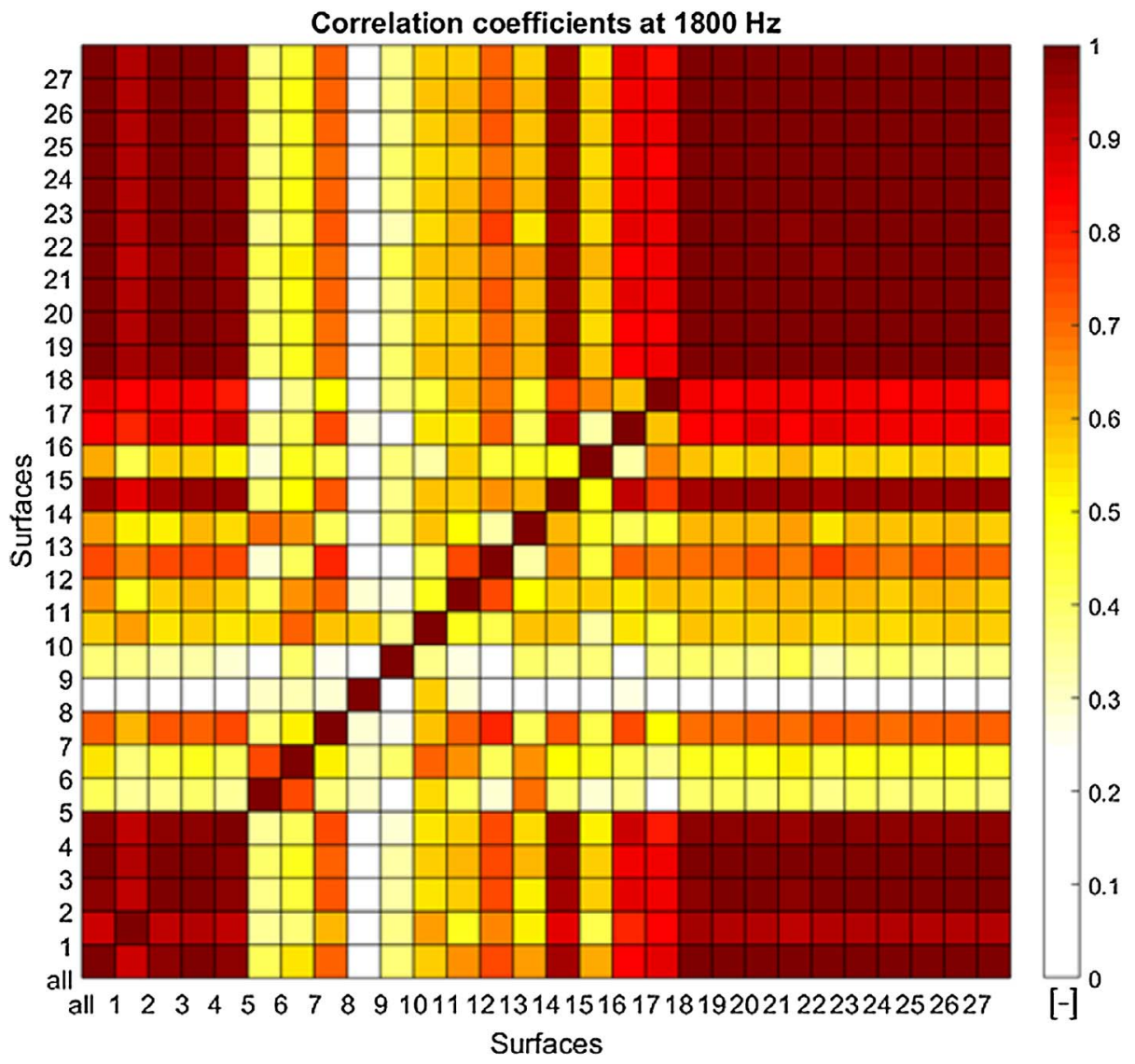

Fig. 13. Pearson Correlation coefficient between acoustic radiated power of different engine surfaces at $1800 \mathrm{~Hz}$.

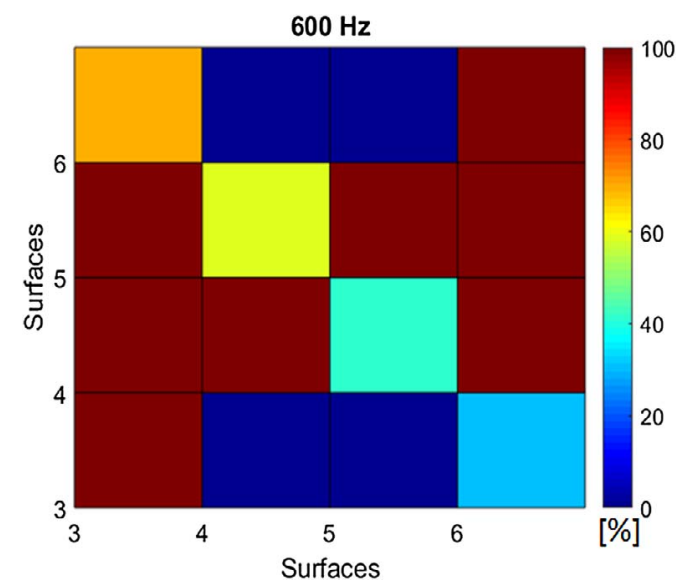

excitation for the analysis on a numerical multi-body systems of the engine tested.

The structural velocities obtained from the multi-body simulation are then used as boundary conditions for the acoustic analysis. A Monte Carlo method is adopted to perform a series of simulations which differ in terms of statistical distribution of the excitation, in accordance with the measured cyclic variability. The results obtained from this analysis are used to derive statistical information on the variability of the results of wave based modeling and on the variability of the noise contribution of the different radiating surfaces. The results obtained show how the variability of the results is in accordance with the variability of the measured cylinder pressure. In general, the variability increases with frequency.

This study also shows how and how much the uncertainties on cylinder pressure influence the ranking of the radiating surfaces. Anyway, the uncertainties arising from results variability can be overcome investigating the cross correlation of the different surfaces contributing to

Fig. 14. Comparisons between power levels radiated by different surfaces at $600 \mathrm{~Hz}$. Data indicate the probability that the contribution on the row is greater or equal compared with the contribution on the column.

\section{Conclusions}

The aim of this paper is to describe a methodology which helps to assess and rank radiating surfaces when excitation uncertainties are considered. In real measurements, excitation variability affects source assessment and ranking. The methodology here introduced helps to quantify this influence. In particular in this paper, the influence of cyclic variability in the exterior acoustic radiation problem using wave based method modeling is investigated. Particular attention is paid on the influence of these excitation uncertainties on the assessment of the different radiating surfaces using a surface contribution analysis. The variability of cylinder pressure is measured from running operating conditions of a test engine and the measured data are used as input the total emitted noise.

\section{Acknowledgements}

The authors gratefully acknowledge the European Commission for their support with the ITN Marie Curie project No. 605867 "BATWOMAN" (Basic Acoustics Training - \& Workprogram on Methodologies for Acoustics - Network). The authors acknowledge the financial support of the COMET K2 - Competence Centres for Excellent Technologies Programme of the Austrian Federal Ministry for Transport, Innovation and Technology (BMVIT), the Austrian Federal Ministry of Science, Research and Economy (BMWFW), the Austrian Research Promotion Agency (FFG), the Province of Styria and the Styrian Business Promotion Agency (SFG). 


\section{References}

[1] Brandl FK, Affenzeller J, Thin GE, Some strategies to meet future noise regulations for truck engines. No. 870950. SAE Technical Paper; 1987.

[2] Boesch NJW. The development of low/noise DI diesel engines. No. 870951. SAE Technical Paper; 1987.

[3] Yuehui L, Zhiyong H, Fengrong B, Lu-hua F, Song-tao H. Engine noise source identification with different methods. Transactions of Tianjin University 2002;8(3):174-7.

[4] Maynard JD, Williams EG, Lee Y. Nearfiled acoustic holography: I. Theory of generalized holography and the development of NAH. J Acoust Soc Am 1985;78(4):1395-413.

[5] Maynard JD, Williams EG, Lee Y. Nearfiled acoustic holography: II. Holographic reconstruction algorithms and computer implementation. J Acoust Soc Am 1987;81(5):1307-22.

[6] Zhang YK, et al., Vehicle noise and weight reduction using panel acoustic contribution analysis. No. 951338. SAE Technical Paper; 1995.

[7] Jahani K, Beigmoradi S, Ramezani A, Hajabdollahi H, Panel contribution analysis for a sedan car using numerical simulations,.In: Proceedings of 20th int congress on sound and vibration (ICSV20), Bangkok, Thailand; 2013.

[8] Mohanty AR, Pierre BDS, Suruli-Narayanasami P. Structure-borne noise reduction in a truck cab interior using numerical techniques. Appl Acoust 2000;59(1):1-17.

[9] Desmet W. A wave based prediction technique for coupled vibro-acoustic analysis. Ph.D. thesis 98D12. Katholieke Universiteit Leuven, Leuven, Belgium; 1998.

[10] Trefftz E. Ein Gegenstück zum ritzschen verfahren. In: Proceedings of the 2nd international congress on applied mechanics, Zürich, Switzerland; 1926, p. 131-7.

[11] Pluymers B, Van Hal B, Vandepitte D, Desmet W. Trefftz-based methods for timeharmonic acoustics. Arch Comput Methods Eng 2007;14:343-81.

[12] Van Genechten B. Trefftz-based mid-frequency analysis of geometrically complex vibro-acoustic problems - hybrid methodologies and multi-level modelling. KULeuven, division PMA. Ph.D. Thesis 2010D08; 2010.

[13] Pluymers B. Wave based modelling methods for steady-state vibro-acoustics. Ph.D. thesis 06D4. Katholieke Universiteit Leuven, Leuven, Belgium; 2006.

[14] Hepberger A, Diwoky F, Jalics K, Priebsch HH. Application of wave based technique for a cavity considering forced excitation at boundaries and effects of absorption materials. In: Proceedings of ISMA, Leuven, Belgium; 2004.

[15] Bergen B, Van Genechten B, Vandepitte D, Desmet W. An efficient wave based method for threedimensional Helmholtz problems in unbounded domains. In: Proceedings of the 16th international congress on sound and vibration (ICSV16), Kraków, Poland; 2009.

[16] van Hal B. Automation and performance optimization of the wave based method for interior structural-acoustic problems. Ph.D. Thesis. Faculty of Engineering, Katholieke Universiteit Leuven; 2004.

[17] Van Genechten B, Atak O, Bergen B, Deckers E, Jonckheere S, Lee JS, et al. An efficient wave based method for solving Helmholtz problems in three-dimensional bounded domains. Eng Anal Bound Elem 2012;36:63-75.

[18] Deckers E, Bergen B, Van Genechten B, Vandepitte D, Desmet W. An efficient wave based method for 2D acoustic problems containing corner singularities. Comput Methods Appl Mech Eng 2012;241-244. 286-30.

[19] Pluymers B, Desmet W, Vandepitte D, Sas P. On the use of a wave based prediction technique for steady-state structural-acoustic radiation analysis. Computer Model Eng Sci 2005;7(2):173-84.

[20] Bergen B, Van Genechten B, Vandepitte D, Desmet W. An efficient Trefftz-based method for three-dimensional Helmholtz problems in unbounded domains. Comput Model Eng Sci 2010;61(2):155-75.

[21] Hepberger A, Priebsch HH, Diwoky F, Pramberger H. Application of the wave based technique to predict the engine noise radiation under anechoic conditions. SAE Int J Passeng Cars-Mech Syst., 2(2009-01-2211); 2009, p. 1477-86.

[22] Acri A, Offner G, Nijman E, Corradi R. Numerical transfer path analysis for the assessment of air-borne noise in internal combustion engines. In: Proceedings of ICoEV 2015 international conference on engineering vibration; 2015, p. 383-92.

[23] Acri A, Offner G, Nijman E, Rejlek J. Substructuring of multibody systems for numerical transfer path analysis in internal combustion engines. Mech Syst Signal Process 2016;79:254-70.

[24] Vanmaele C, Vandepitte D, Desmet W. An efficient wave based prediction technique for plate bending vibrations. Comput Methods Appl Mech Eng 2007;196:3178-89.

[25] Vanmaele C, Vandepitte D, Desmet W. An efficient wave based prediction technique for dynamic plate bending problems with corner stress singularities. Comput Methods Appl Mech Eng 2009;198:2227-45.

[26] Vergote K, Vanmaele C, Vandepitte D, Desmet W. An efficient wave based approach for the time-harmonic vibration analysis of 3D plate assemblies. J Sound Vibrat 2013;332:1930-46.

[27] Klanner M, Ellermann K. Wave based method for the steady-state vibrations of thick plates. J Sound Vibrat 2015;345:146-61.

[28] Klanner M, Ellermann K. Improvement of the wave based method for thick plate vibrations. Int J Acoust Vibrat; 2016.

[29] Lanoye R, Vermeir G, Lauriks W, Sgard F, Desmet W. Prediction of the sound field above a patchwork of absorbing materials. J Acoust Soc Am 2008;123:793-802

[30] Deckers E, Hörlin N-E, Vandepitte D, Desmet W. A wave based method for the efficient solution of the 2D poroelastic Biot equations. Comput Methods Appl Mech
Eng 2012;201-204:245-62.

[31] Deckers E, et al. The wave based method: an overview of 15 years of research. Wave Motion 2014;51(4):550-65.

[32] Acri A, Zsifkovits P, Nijman E, Offner G, Corradi R. Surface noise contribution analysis in wave based method framework for exterior radiation noise problems. In: Proceedings of ISMA, Leuven, Belgium; 2016.

[33] Offner G, Priebsch HH. Flexible multi-body dynamics simulation - a powerful method for prediction of structure borne noise of internal combustion engines. In: Proceedings of ISMA2006, Leuven Belgium, 18-20; September 2006.

[34] Rahnejat H. Multi-body dynamics: historical evolution and application. Proc Inst Mech Eng Part C: J Mech Eng Sci 2000;214(1):149-73.

[35] Shabana AA. Flexible multibody dynamics: review of past and recent developments. Multibody Syst Dyn 1997;1(2):189-222.

[36] Bauchau OA. Flexible multibody dynamics, vol. 176. Springer Science \& Business Media; 2010.

[37] Rubinstein RY. Simulation and the monte carlo method. New York: John Wiley; 1981.

[38] Spanos PD, Mignolet MD. Arma Monte Carlo simulation in probabilistic structural analysis. Shock Vibrat Digest 1989;21:3-10.

[39] Evensen G. Sequential data assimilation with a nonlinear quasi-geostrophic model using Monte-Carlo methods to forecast error statistics. J Geophys Res 1994;99(C5).

[40] McKay MD, Beckman RJ, Conover WJ. A comparison of three methods for selecting values of input variables in the analysis of output from a computer code. Technometrics 1979;21(2):237-40.

[41] Bergin MS, Noblet GS, Petrini K, Dhieux JR, Milford JB, Harley RA. Formal uncertainty analysis of a lagrangian photochemical air pollution. Model Environ Sci Technol 1999;32:1116-26.

[42] Bergin MS, Milford JB. Application of bayesian Monte Carlo analysis to a lagrangian photochemical air quality model. Atmos Environ 2000;33(5):781-92.

[43] Clerk D. The gas engine. 1st ed. London: Longmans, Green \& Co; 1886

[44] Patterson DJ. Cylinder pressure variations, a fundamental combustion problem. No. 660129. SAE Technical Papers; 1966

[45] Young MB. Cyclic dispersion in the homogeneous-charge spark-ignition engine - a literature survey. No. 810020. SAE Technical papers; 1981.

[46] Ozdor N, Dulger M, Sher E, Cyclic variability in spark-ignition engines: a literature survey. No. 940987. SAE Technical papers; 1994.

[47] $\mathrm{Hu} \mathrm{Z}$. Nonlinear instabilities of combustion processes and cycle-to-cycle variations in spark ignition engines. No. 961197. SAE Technical papers; 1996.

[48] Chew L, Hoekstra R, Nayfeh JF, Navedo J. Chaos analysis of in-cylinder pressure measurements. No. 942486,.SAE Technical papers; 1994.

[49] Daily JW. Cycle-to-cycle variations: a chaotic process? Combust Sci Technol 1988;57:149-62.

[50] Sen AK, Longwic R, Litak G, Górski K. Analysis of cycle-to-cycle pressure oscillations in a diesel engine. Mech Syst Signal Process 2008;22(2):362-73.

[51] Finney CE, Kaul BC, Daw CS, Wagner RM, Edwards KD, Green JB. A review of deterministic effects in cyclic variability of internal combustion engines. Int $\mathrm{J}$ Engine Res 2015;16(3):366-78.

[52] Pierce A. Acoustics: an introduction to its physical principles and applications. In: McGraw-Hill Series in Mechanical Engineering, McGraw-Hill; 1981.

[53] Sommerfeld A. Partial differential equations in physics. New York: Academic Press 1949.

[54] Rejlek J, Mocsai T, Silar P, Hepberger A, Priebsch HH. Wave based prediction technique for sound radiation analysis. In: Proceedings of international conference on acoustics, NAG/DAGA; 2009, p. 23-6.

[55] Jegorovs J. On the convergence of the WBM solution in certain nonconvex domains. In: Proceedings of the international conference on noise and vibration engineering ISMA2006, Leuven, Belgium; 2006, p. 2201-9.

[56] Bergen B. Wave based modelling techniques for unbounded acoustic problems. $\mathrm{Ph}$. D. Thesis. Katholieke Universiteit Leuven; 2011.

[57] Pluymers B, Desmet W, Vandepitte D, Sas P. Application of an efficient wave-based prediction technique for the analysis of vibro-acoustic radiation problems. J Comput Appl Math 2004;168(1):353-64.

[58] AVL LIST GmbH: AVL-excite reference manual. version 2016.

[59] Ross SM. Introduction to probability and statistics for engineers and scientists. Academic Press; 2014.

[60] Priede T. Noise and engineering design. Philosop Trans Royal Soc London Series A Math Phys Sci 1968;263(1142):461-80

[61] Offner G. Modelling of condensed flexible bodies considering non-linear inertia effects resulting from gross motions. Proc Inst Mech Eng Part K: J Multi-body Dyn 2011;225(3):204-19.

[62] Gerstmayr J, Ambrosio JAC. Component mode synthesis with constant mass and stiffness matrices applied to flexible multi-body systems. Int J Numer Methods Eng 2008;73:1518-46.

[63] Guyan RJ. Reduction of stiffness and mass matrices, AIAA J (3); February 1965.

[64] Nijman E. Airborne sound of automotive IC engines: a piece of cake or a can of worms?. In: Proceedings of 33. Deutsche Jahrestagung für Akustik - DAGA 2007, Stuttgart; March 2007.

[65] Pearson K. Notes on regression and inheritance in the case of two parents. Proc Royal Soc London 1985;58:240-2. 